\title{
Extended Spectrum $\beta$-Lactamases among Gram-Negative Bacterial Isolates from Clinical Specimens in Three Major Hospitals in Northern Jordan
}

\author{
Raymond G. Batchoun, Samer F. Swedan, and Abdullah M. Shurman \\ Department of Medical laboratory Sciences, Faculty of Applied Medical Sciences, Jordan University of Science and Technology, \\ P.O. Box 3030 Irbid 22110, Jordan \\ Correspondence should be addressed to Raymond G. Batchoun, batchounr@yahoo.com
}

Received 21 May 2009; Accepted 25 July 2009

Recommended by William M. Shafer

Background and Objectives. Extended spectrum $\beta$-lactamase (ESBL) production is increasing all over the world, and organisms other than E. coli and K. pneumoniae are acquiring this character. ESBL production is detectable by automation, E-test, double disk diffusion (DDD), and PCR. This study aimed to determine the prevalence of ESBL production among clinical isolates of gram-negative rods, and to evaluate the effectiveness of augmentation of clavunate with Cefotaxime, Ceftazoxime, Aztreonam, Ceftriaxone, and Cefpodoxime in detecting ESBL production. Methods. 472 clinical gram-negative isolates identified by standard methods were tested for ESBL-production by (DDD) method using six cephalosporins and amoxicillin-clavulinate discs. Results. $108 / 472(22.9 \%)$ of the isolates were ESBL producers, and were prevalent in tertiary care hospitals. $88.2 \%$ of E. cloacae, $71.4 \%$ of $K$. pneumoniae, $28.6 \%$ of $K$. oxytoca, $12.5 \%$ of $C$. freundii, $11.1 \%$ of A. calcoacceticus, and $10.8 \%$ of E. coli were ESBL producers. The DDD test demonstrated some variations in the efficacy of the different cephalosporins in detecting all the ESBL producers. The inclusion of ceftizoxime discs increased the efficacy of the test. It is concluded that ESBL-producing bacteria were prevalent among our hospitalized patients, and involved genera other than Klebsiella and Escherichia, and the inclusion of ceftizoxime increased the efficacy of ESBL detection by the DDD test.

Copyright (C) 2009 Raymond G. Batchoun et al. This is an open access article distributed under the Creative Commons Attribution License, which permits unrestricted use, distribution, and reproduction in any medium, provided the original work is properly cited.

\section{Introduction}

Among the family Enterobacteriacae, the production of plasmid-mediated extended-spectrum $\beta$-lactamase (ESBLs) has emerged as an important mechanism of resistance to $\beta$-lactam drugs that account for approximately $50 \%$ of antibiotic consumption [1]. The vast majority of ESBLs are derivatives of TEM-1 and TEM-2 chromosomally encoded enzyme of Klebsiella pneumoniae [2]. These enzymes are capable of hydrolyzing a wide range of $\beta$-lactams, including most recently developed cephalosporins, but are not active against cephamycins and carapenems [3]. In interpreting the phenotype of ESBL-positive strains with regard to $\beta$-lactams, it should be borne in mind that drug resistance may also result from the combined activity of a specific ESBL together with other $\beta$-lactamases (the chromosomal AmpC) [3], or the plasmid-borne one [4].
Among Enterobacteriacae, ESBLs have been found mainly in Klebsiella spp. and Escherichia coli, but have been also reported in other genera world wide, such as Citrobacter, Enterobacter, Morganella, Proteus, Providencia, Salmonella, Serrati, and Pseudomonas [5-9].

Infections caused by ESBL-producing bacteria often involve immune-compromised patients, making it difficult to eradicate these organisms in high-risk wards, such as intensive care unites [10,11].

Microbiology laboratories play an important role in detecting and promptly reporting the isolation of ESBL-positive bacteria, especially AmpC beta-lactamasesproducing ones that complicate therapy and limit treatment options [12]. Drug susceptibility data are of major importance for the clinical management of patients infected by these organisms [13]. Reduced susceptibility or increase in resistance to extended-spectrum cephalosporins and/or 
monobactams represents the first indicator of ESBL production, but confirmation is dependent on synergy between clavulanate and the selected $\beta$-lactams, using doubledisk diffusion method, or E-test [14]. The expression of an extended-spectrum enzyme does not always involve a phenotype that can be interpreted as resistant by the routine MICs and disk diffusion methods that follow current National Committee for Clinical Laboratory Standards/recently named Clinical and Laboratory Standards Institute (NCCLS/CLSI) breakpoint. Accordingly, ESBLpositive strains should be reported as resistant even if drug MICs are below breakpoints established for cephalosporins and aztreonam. This is defined for both Klebsiella spp, and E.coli, but not established for the other Enterobacteriaceae [15].

When detecting ESBL-positive strains, microbiology laboratories should provide the clinician with reliable therapeutic options for successfully treating infected patients, since ESBL-distribution has been shown to differ among countries $[3,6,16]$. Thus monitoring of the prevalence and the types of extended spectrum $\beta$-Lactamase enzymes may contribute to defining the degree of the problem in a specific geographical area, and establishing a proper treatment protocol.

Before the emergence and increased spread of ESBLproducing gram-negative bacteria, most infections could be reliably treated with second- and third-generation cephalosporins. However, ESBL-producing organisms are spreading world wide, resulting in the failure of empiric therapy dependent on second- and third-generation cephalosporins which complicate infections in immunecompromised patients, neonates, the elderly, debilitated patients, nosocomial infections, and outbreaks occurring in hospital setting [17-22].

The aim of this study was to assess the prevalence of ESBL-producing gram-negative organisms among different bacterial genera and species isolated from clinical cases from three major hospitals in northern Jordan, and to assess the effectiveness of clavulanate and six cephalosporins in detecting ESBL production.

\section{Materials and Methods}

2.1. Bacterial Isolates. A number of 472 gram-negative bacterial isolates recovered from 463 patients were included in this study. These isolates were isolated from clinical specimens at the microbiology laboratories in three hospitals in northern Jordan during ten-month period (JanuaryOctober 2004). These hospitals were King Abdullah University Teaching Hospital (KAUTH) (tertiary care hospital), Princess Basma Teaching Hospital (PBTH) (primary and secondary care hospital), and Princess Rahmeh Teaching Hospital (PRTH) (secondary care hospital dealing with pediatrics, neonates, obstetrics and gynecology, and immunesuppressed patients). Gram-negative bacteria belonging to the genera Escherichia, Klebsiella, Proteus, Citrobacter, Enterobacter, Serratia, Pseudomonas, Acinetobacter, Alkaligenes, and Stenotrophemonas were included in the study. These bacteria were isolated from various clinical specimens such as blood culture, urine, CSF, sputum, wound, pus, ear swabs, eye swabs, peritoneal fluid, and other miscellaneous sources. These isolates were recovered from the specimens by being cultured on the specific media, under the optimal conditions, and were identified to genus and species using standard methods (conventional manual methods, Rapid-ID—Remel, USA, and Vitek automation technology) [23-25]. Multiple samples or isolates from the same patient were excluded from the study. These isolated were stored on simple storage media as described by Evans et al. in 1977 [26].

2.2. ESBL Production. Test organisms from stock culture were activated by inoculation in to Mueller Hinton broth (BD, USA) and incubated at $37^{\circ} \mathrm{C}$ for 24 hours. The concentration of the bacterial suspension was adjusted to be equivalent to $0.5 \mathrm{McF}$ arland standards. The test organism was seeded on the surface of freshly prepared Mueller Hinton agar (BD, USA), in three directions using a sterile Dacron swab, according to the recommendations of KirbyBauer Disk Diffusion method [25], and NCCLS (CLSI) guide lines [27]. The plates were allowed to stand at room temperature for 15 minutes prior to the application of antibiotic containing disks.

ESBL-producing isolates were detected using the Double Disk Diffusion method (Double Disk Approximation Test) [28]. Six antibiotic disks (Cefotaxime $30 \mathrm{ug}$, Ceftazidime 30 ug, Ceftriaxone 30 ug, Cefpodoxime 10 ug, Ceftizoxime $30 \mathrm{ug}$, and Aztreonam $30 \mathrm{ug}$ ) (Oxoid, UK) were placed around a central disk of Amoxicillin-Clavulanic acid $30 \mathrm{ug}$ (20:10 resp.) (Oxoid, UK), $30 \mathrm{~mm}$ center to center on Mueller Hinton agar plates seeded with organism being tested for ESBL production.

Plates were incubated aerobically at $37^{\circ} \mathrm{C}$ for $18-24$ hours, and the diameter of the inhibition zone (if any) around the antimicrobial disks was measured in mm using a ruler. Any augmentation (increase in diameter of inhibition zone) between the central Amoxicillin-Clavulanic acid disk and any of the six antibiotic disks showing resistance or intermediate susceptibility was recorded, and the organism was thus considered as an ESBL producer.

\section{Results}

3.1. Distribution of Bacterial Species with Sample Source. Urine was the major source of the bacterial isolates collected, comprising 262/472 (56\%) of the total isolates, blood culture $79 / 472(16.7 \%)$, swabs from various sites $80 / 472$ (16.9\%), CSF $16 / 472(3.3 \%)$, sputum $8 / 472(1.6 \%)$, and the other miscellaneous sources 44/472 (5.5\%) (Table 1).

Escherichia coli was the most common species isolated from these specimens, comprising 195/472 (41.4\%). This organism was the major isolate recovered from urine samples, representing $171 / 195$ (87.7\%) of the total E. coli isolates from the three teaching hospitals (Table 1).

Genus Klebsiella was the second isolated pathogen from the tested samples, constituting 132/472 (28\%) of the total isolates, of which Klebsiella pneumonia represented 84/472 $(17.8 \%)$ of the total isolates. However, $48 / 84(57 \%)$ of the 
TABLE 1: Bacterial species isolated from clinical samples.

\begin{tabular}{|c|c|c|c|c|c|c|c|}
\hline Bacterial species & $\begin{array}{l}\text { Blood } \\
\text { culture }\end{array}$ & CSF & Sputum & Swab & Urine & Miscellaneous & Tota \\
\hline Acinetobacter baumannii & & & & & & 2 & 2 \\
\hline Acinetobacter calcoaceticus & 6 & & 1 & & & 2 & 9 \\
\hline Acinetobacter lwoffii & 1 & & & & & & 1 \\
\hline Acinetobacter species & & & 1 & 2 & 1 & 2 & 6 \\
\hline Alcalgenes xylosoxidans & & 1 & & & & & 1 \\
\hline Citrobacter frundii & 1 & & & 1 & 6 & & 8 \\
\hline Escherichia coli & 6 & 1 & & 13 & 171 & 4 & 195 \\
\hline Enterobacter aerogenes & & & & & 1 & & 1 \\
\hline Enterobacter cloacae & 11 & & 1 & & 5 & & 17 \\
\hline Enterobacter hormaechei & & & & & 1 & 1 & 2 \\
\hline Enterobacter species & & & & 7 & 4 & 1 & 12 \\
\hline Klebsiella pneumoniae & 1 & & & 8 & 19 & & 28 \\
\hline Klebsiella oxytoca & 48 & 1 & 2 & 9 & 21 & 3 & 84 \\
\hline Proteus mirabilis & & & & 6 & 16 & 1 & 23 \\
\hline Pseudomonas aeruginosa & 4 & 12 & 3 & 24 & 10 & 11 & 64 \\
\hline Pseudomonas species & & 1 & & 10 & 4 & 1 & 16 \\
\hline Serratia marcescens & & & & & 1 & & 1 \\
\hline $\begin{array}{l}\text { Stenotrophemonas } \\
\text { maltophilia }\end{array}$ & & & & & 2 & & 2 \\
\hline Total & 79 & 16 & 8 & 80 & 262 & 27 & 472 \\
\hline
\end{tabular}

Miscellaneous: Aspirate, Bone, Cornea, Peritoneal, Tissues, Tracheostomy.

Klebsiella pneumoniae isolates were recovered from blood culture, 21/84 (25\%) from urine, and the remaining 15/84 (18\%) from sputum and the other miscellaneous sources. Similarly, Klebsiella oxytoca comprised 28/472 (5.9\%) of the total isolates, of which 19/28 (67.9\%) were isolated from urine, and the remaining 9/28 (32.1\%) were detected at low frequency from the specimens (Table 1).

Pseudomonas aeruginosa represented 64/472 (13.5\%) of the total isolates, where $24 / 64$ (37.5\%) of these pseudomonas aeruginosa were isolated from swabs of various sources, 12/64 (18.8\%) from Cerebrospinal fluid (CSF), 10/64 (15.6\%) from urine, and the remaining 18/64 (28.1\%) were from the other miscellaneous sources (Table 1). Additionally, 16/472 (3.4\%) pseudomonas spp other than Pseudomonas aeruginosa were isolated mainly from various swabs, urine, and the other miscellaneous samples.

Proteus mirabilis isolates represented 23/472 (4.8\%) of the bacterial isolates, where $16 / 23(69.5 \%)$ of them were isolated from urine samples, $7 / 23$ (30.4\%) from swabs of various sources and other miscellaneous specimens (Table 1).

Genus Enterobacter was common among the gramnegative isolates constituting 32/472 (7.8\%) of the isolates. Enterobacter cloacae was the major species isolated constituting 11/17 (64.7\%) of the Enterobacter recovered from blood cultures, 5/17 (29.7\%) from urine cultures, and 1/17 (5.9\%) from sputum samples. The other 14 Enterobacter spp were isolated from swabs of various sources and urine (Table 1).

Bacterial isolates belonging to the genus Acinetobacter (Acinetobacter baumannii, Acinetobacter calcoaceticus,
Acinetobacter lwoffii, and other Acinetobacter spp) constituted 18/472 (3.8\%) most of which were recovered from blood culture, sputum, and miscellaneous sources. Other gram-negative bacterial species, including Citrobacter frundii, Serratia marcescens, Alkaligenes xylosoxidans, and Stenotrophomonas maltophilia, collectively represented $12 / 472(2.5 \%)$, most of which were isolated from urine samples (Table 1$)$.

3.2. ESBL Production. The Double Disk diffusion test revealed that out of the 472 gram-negative isolates included in the study, 108 isolates were ESBL producers. These ESBLproducing bacteria belonged to 8 different species out of the 18 species isolated from the clinical samples from the three teaching hospitals (Table 2).

ESBL production was very common among Klebsiella pneumoniae, where out of the 84 Klebsiella pneumoniae isolates, 49 (71.4\%) were ESBL producers. However, within Klebsiella oxytoca isolates, only 8 out of 28 isolates were ESBL-producers, representing only $28.5 \%$. On the other hand, ESBL production in E. coli was found in 21/195 isolates representing only $10.8 \%$ (Table 2 ).

Out of the 17 Enterobacter cloacae isolated, 15 (88.2\%) were ESBL producers, and all of them were recovered from blood culture and urine culture from KAUTH during Enterobacter cloacae outbreak (Table 2).

ESBL production in Citrobacter frundii was found only in one isolate out of the 8 isolates (12.5\%) recovered from 
TABLE 2: ESBL production among the gram-negative bacteria.

\begin{tabular}{lcccccccc}
\hline & $\begin{array}{c}\text { Acinetobacter } \\
\text { calcoaceticus }\end{array}$ & $\begin{array}{c}\text { Citrobacter } \\
\text { frundii }\end{array}$ & $\begin{array}{c}\text { Escherichia } \\
\text { coli }\end{array}$ & $\begin{array}{c}\text { Enterobacter } \\
\text { cloacae }\end{array}$ & $\begin{array}{c}\text { Enterobacter } \\
\text { hormaechei }\end{array}$ & $\begin{array}{c}\text { Klebsiella } \\
\text { oxytoca }\end{array}$ & $\begin{array}{c}\text { Klebsiella } \\
\text { pneumoniae }\end{array}$ & $\begin{array}{c}\text { Stenotrophemonas } \\
\text { maltophilia }\end{array}$ \\
\hline $\begin{array}{l}\text { Total isolate } \\
\text { Number ESBL }\end{array}$ & 9 & 8 & 195 & 17 & 2 & 28 & 84 & 2 \\
$\begin{array}{l}\text { producer } \\
\begin{array}{l}\text { Percentage ESBL } \\
\text { producer/species }\end{array}\end{array}$ & 11.1 & 1 & 21 & 15 & 1 & 8 & 60 & 1 \\
\hline
\end{tabular}

KAUTH. Similarly, only one Acinetobacter calcoaceticus out of the nine isolates was ESBL producer. None of the other Acinetobacter spp. isolated produced ESBL (Table 2). Further more, one isolate of both Enterobacter hormaechei and Stenotrophemonas maltophilia was ESBL producer (Table 2). Most of the ESBL-producing isolates were recovered from patients samples collected from the tertiary care KAUTH.

3.3. Effectiveness of Antibiotics Used in Detecting ESBL Production. The most important feature in detecting ESBLproducing isolates by the Double Disk Diffusion method is the formation of augmentation of the bacterial growth inhibition zone between the central Amoxicillin-Clavulanic acid (AMC) disk and the surrounding cephalosporin's ones. The 21 ESBL-producing Escherichia coli isolates gave the highest number of augmentation zones when Cefotaxime (CTX) and Ceftizoxime (ZOX) disks were used. Both of these antibiotics were successful in identifying 20/21 (95.2\%) of the ESBL producers. Augmentation with Aztreonam (ATM) detected 18/21 (85.7\%) ESBL-producing E. coli, Ceftazidime (CAZ) 15/21 (71.4\%), Ceftriaxone (CRO) 11/21 (52.4\%), and Cefpodoxime (CPD) 4/21 (19\%) (Table 3).

ESBL-producing Enterobacter cloacae, which were collected during an out break in KAUTH, had little variation in the antibiogram of the isolates suggesting a common source. Disks used in the detection of ESBL were closely successful in detecting ESBL production, where ATM was capable of detecting all of the $15 \mathrm{ESBL}$ producing isolates, followed by CTX and CRO which detected 14/15 (93.3\%) of them. Both CAZ and ZOX detected only 13/15 (86.7\%), while CPD detected only $12 / 15$ (80\%) of the ESBL-producing Enterobacter cloacae (Table 3 ).

Klebsiella pneumoniae isolates producing ESBL gave augmentation with ZOX, where it detected 57/60 (95\%) of the ESBL-producing isolates, CTX 49/60 (81.7\%), ATM 43/60 (71.7\%), CAZ 30/60 (60\%), CRO 26/60 (43.3\%), and CPD 15/60 (16.7\%), respectively, (Table 3). Similarly, out of the 8 ESBL-producing Klebsiella oxytoca, ZOX detected $7 / 8$ $(87.5 \%)$ of the ESBL producers, ATM 6/8 (75\%), CTX 4/8 (50\%), CAZ 3/8 (37.5\%), and CRO 2/8 (25\%), respectively, (Table 3).

On the contrary, effectiveness of the antibiotics used in the detection of ESBL production in Acinetobacter calcoaceticus, Citrobacter frundii, Enterobacter hormaechei, and Stenotrophemonas maltophilia could not be established due to the small number of ESBL-producing isolates among these species (Table 3 ).

\section{Discussion}

This study included three major hospitals in northern Jordan, to assess the prevalence and distribution of ESBLpositive species among Enterobacteriaceae and other gramnegative bacteria recovered from clinical specimens, and evaluated the efficacy of different cephalosporins in the detection of ESBL production.

In a study between 1990 and 1993, Youssef et al., in 1999 reported that 38\% of Klebsiella pneumoniae isolates were ESBL producers [29]. Shehabi et al., in 1999 reported an incidence of ESBL production in Klebsiella pneumoniae and Escherichia coli isolates in the ICU of Jordan University Hospital, to be $70 \%$ and $38 \%$, respectively, [30]. Batchoun and Matalka (unpublished data) did not detect any ESBL producers among various pseudomonas species.

Extended-spectrum $\beta$-lactamases (ESBLs) are enzymes capable of hydrolyzing oxyimino-cephalosporins, such as Cefotaxime (CTX), Ceftriaxone, Ceftazidime (CAZ), and monobactams (e.g., aztreonam (ATM)), thereby causing resistance to these drugs. The enzymes are detected most commonly in Klebsiella pneumoniae and Escherichia coli but have been noted in other members of the family Enterobacteriaceae as well [22]. The majority of these enzymes usually have only 1,2 , or 3 amino acid mutation from those of the parent enzymes (TEM-1, TEM-2, and SHV-1). These mutations are thought to have evolved under selective pressures exerted by antibiotic treatment, and continued use of cephalosporin antibiotics. The fact that most of these enzymes are carried by plasmids has facilitated the spread of ESBL enzymes among members of the family Enterobacteriaceae and other gram-negative bacteria, and single strain of K. pneumoniae or E. coli may harbor different variants of ESBL genes such as SHV with CTX-M type, AmpC or metalo- $\beta$-lactamases together, that may complicate therapy [31].

In our study, urine was the source of 262/472 (56\%) of the isolates, indicating that UTI is a common illness in our community. Blood culture $(16.7 \%, 79 / 472)$ was the second major source of isolates thus indicating the relatively high frequency of gram-negative bacteria involved in bacteremias or septicemias in hospitalized patients. The rest of the isolates collected in our study were from ear swabs, swabs from miscellaneous sources, CSF, and sputum.

Out of the 472 isolates recovered in this study, 195 isolates were E. coli and 84 were K. pneumoniae. Both E. coli and $K$. pneumoniae are involved world wide in ESBL production, however, as major isolates from our hospitals, they can give us a good picture about ESBL production in our community. 
TABLE 3: Efficasy of cephalosporins in detecting ESBL-producers.

\begin{tabular}{|c|c|c|c|c|c|c|c|c|c|c|c|c|}
\hline Species (count) & Interpretation & AMC & CTX & CAZ & ATM & $\mathrm{CRO}$ & ZOX & $\mathrm{CPD}$ & CTT & FEP & $\mathrm{CXM}$ & CFP \\
\hline \multirow{4}{*}{ Acinetobacter calcoaceticus (1) } & $S$ & & 1 & & & & 1 & & 1 & 1 & & \\
\hline & I & & & & & 1 & & & & & & 1 \\
\hline & $\mathbf{R}$ & 1 & & 1 & 1 & & & 1 & & & 1 & \\
\hline & A & & 1 & 1 & 1 & 1 & 1 & 1 & & 1 & 1 & 1 \\
\hline \multirow{4}{*}{ Citrobacter frundii (1) } & $S$ & & & & & & & & 1 & & & \\
\hline & I & & & & & & & & & & & \\
\hline & $\mathbf{R}$ & 1 & 1 & 1 & 1 & 1 & 1 & 1 & & 1 & 1 & 1 \\
\hline & A & & 1 & & & & 1 & & & 1 & & \\
\hline \multirow{4}{*}{ Escherichia coli (21) } & $S$ & 7 & & 6 & & & 8 & & 21 & 4 & & \\
\hline & I & 11 & 3 & 4 & 4 & 3 & 9 & & & 6 & 1 & 1 \\
\hline & $\mathbf{R}$ & 3 & 18 & 11 & 17 & 18 & 4 & 21 & & 11 & 20 & 20 \\
\hline & A & & 20 & 15 & 18 & 11 & 20 & 4 & 7 & 18 & 4 & 11 \\
\hline \multirow{4}{*}{ Enterobacter cloacae (15) } & $\mathbf{S}$ & & & & & & 12 & & 3 & 12 & & 1 \\
\hline & I & & 12 & 1 & & 12 & 2 & & 1 & 1 & & 11 \\
\hline & $\mathbf{R}$ & 15 & 3 & 14 & 15 & 3 & 1 & 15 & 11 & 2 & 15 & 3 \\
\hline & A & & 14 & 13 & 15 & 14 & 13 & 12 & 1 & 15 & 12 & 13 \\
\hline \multirow{4}{*}{ Enterobacter hormaechei (1) } & $S$ & & & & & & & & & & & \\
\hline & I & & & & & & 1 & & & & & \\
\hline & $\mathbf{R}$ & 1 & 1 & 1 & 1 & 1 & & 1 & & 1 & 1 & 1 \\
\hline & A & & & & & & 1 & & & & & \\
\hline \multirow{4}{*}{ Klebsiella oxytoca (8) } & $S$ & & & 1 & & & 2 & & 7 & 1 & & \\
\hline & I & 5 & & 1 & 1 & & 3 & & 1 & 1 & & \\
\hline & $\mathbf{R}$ & 3 & 8 & 6 & 7 & 8 & 3 & 8 & & 6 & 8 & 8 \\
\hline & A & & 4 & 3 & 6 & 2 & 7 & & & 6 & & 4 \\
\hline \multirow{4}{*}{ Klebsiella pneumonia (60) } & $S$ & 8 & & 27 & 1 & & 31 & & 60 & 17 & 1 & \\
\hline & I & 37 & 16 & 9 & 2 & 15 & 29 & & & 8 & 5 & 9 \\
\hline & $\mathbf{R}$ & 15 & 44 & 24 & 57 & 49 & & 60 & & 35 & 54 & 51 \\
\hline & A & & 49 & 36 & 43 & 26 & 57 & 15 & 10 & 46 & 11 & 31 \\
\hline \multirow{4}{*}{ Stenotrophemonas maltophilia (1) } & $S$ & 1 & 1 & 1 & & & & & 1 & 1 & & 1 \\
\hline & I & & & & 1 & 1 & & & & & & \\
\hline & $\mathbf{R}$ & & & & & & 1 & 1 & & & 1 & \\
\hline & A & & & & 1 & 1 & 1 & 1 & & & & \\
\hline
\end{tabular}

AMC: Amoxicillin-Clavulanic Acid, CTX: Cefotaxime, CAZ: Ceftazidime, ATM: Aztreonam, CRO: Ceftriaxone, ZOX: Ceftizoxime, CPD: Cefpodoxime, CTT: Cefotetan, FEP: Cefepime, CXM: Cefuroxime, and CFP: Cefoperazone, S: Sensitive, I: Intermediate, R: Resistant, A: Augmentation.

Other clinically important isolates that include Pseudomonas aeruginosa (64/472), Klebsiella oxytoca (28/472), Proteus mirabilis (23/472), Enterobacter cloacae (17/472), and Pseudomonas Spp (16/472) were recovered in moderate number, whereas the remaining isolates were scarce. Although the moderate number of these isolates is high enough to allow a calculation of the percentage of ESBL production, this percentage may not actually reflect the true nature of ESBL production in the isolate nation wide, and may only indicate the presence of an endemic bacteria in a specific hospital or a specific ward. This is certainly true-if not at least-for the 17 isolates of Enterobacter cloacae which were all collected from King Abdullah University Teaching Hospital during an outbreak.

In regard to ESBL production, one of the most alarming findings of this study is the ESBL production in 60/84 $(72.4 \%)$ of the Klebsiella pneumoniae isolates. This percent- age is considered to be very high compared to prevalence of ESBL production world wide among this species when compared with the $20 \%$ prevalence of the Italian study [32], and $39.5 \%$ in the Chinese study [33], 11.3\% in Saudi Arabian study [34], and $13.3 \%$ in the Kuwaiti study [35]. However, our results are matching previous Jordanian study which reported that $70 \%$ of the K. pneumoniae isolates recovered from the ICU of Jordan University Hospital to be ESBL producers [30]. On the other hand, none of the Klebsiella pneumoniae isolates recovered from Princess Basma Teaching Hospital were ESBL producer, which may be attributed to the low number of isolates recovered, the nature of the hospital type, and the patients being served. On the contrary, Isolates from Princess Rahma Teaching Hospital and King Abdullah University Teaching Hospital showed ESBL production in 35/39 (89.7\%) and 25/32 (78.1\%), respectively. The abnormally high percentage of ESBL production in 
these two hospitals may indicate the presence of a previously undetected source of a nosocomial infection, the nature of patients being served as both hospitals are serving debilitated patients, and as referral hospitals for malignancy and chronic diseases. Such findings impose the need for applying specific infection control measures to eliminate this organism.

One of the highest ESBL producers by percentage was found to be Enterobacter cloacae, which was found in 15/17 collected isolates, thus constituting $88.2 \%$. As indicated before, this high percentage disagrees with prevalence of ESBL production in this species which was $2.9 \%$ in the Italian study [32], 6\% in the Chinese study [33], and $12.8 \%$ in the Korean study [8]. This observation could only be explained on the fact that all these Enterobacter cloacae isolates were recovered from King Abdullah University Teaching Hospital during a period of outbreak, as supported by their semiidentical antibiogram profiles and DDD test results.

In Escherichia coli isolates, ESBL production was found in 21 out of a total of 195 isolates recovered (10.8\%). This percentage agrees with the Chinese study, which demonstrated ESBL production in $11.4 \%$ of their Escherichia coli isolates [33], the Saudi Arabian study (9.6\%) [34], and the Kuwaiti study $(11.7 \%)$ [35], but higher than that of the Italian study $(1.2 \%)$ [32], and lower than the Taiwanese one [11]. It is worth noting that most ESBL-producing E. coli isolates were from King Abdullah University Teaching Hospital, whereas those from PBTH and PRTH did not exceed $6 \%$.

In Klebsiella oxytoca isolates, ESBL production was found in $8 / 28(28.5 \%)$ of the isolates recovered. This percentage (although the sample size is not large enough) is considered to be high when compared to those for Klebsiella oxytoca worldwide, where the Italian study reported $15 \%$ prevalence [32]. It is also worth noting that isolates from KAUTH showed the highest degree of ESBL production $(80 \%$, 4/5), whereas PRTH and PBTH showed ESBL production in $2 / 9$ isolates $(22.2 \%)$, and $2 / 14(14.3 \%)$, respectively. Furthermore, ESBL started to appear among Citrobacter frundii, Acinetobacter calcoaceticus, Enterobacter hormaechei, and Stenotrophemonas maltophilia isolates from the tertiary care hospital KAUTH, which may reflect the expansion of the genes coding for ESBLs production to other bacterial genera. Although their prevalence in our samples is not high, however, these organisms are becoming a threat to ICU patients and becoming involved in nosocomial infections as reported in Taiwanese, Bulgarian, and Korean hospitals $[8,9,11]$.

The NCCLS (CLSI) [27] recommend testing for ESBL production using CTX, CAZ, ATM, CRO, and CPD in combination with AMC. In our study, we have evaluated the effectiveness of each of the above antibiotics in addition to ZOX in detection of ESBL production by the DDD method.

In Escherichia coli, both CTX and ZOX detected 20/21 (95\%) of the ESBL-producing isolates, whereas ATM detected only $85.7 \%$ of the isolates. However, CAZ, CRO, CPD were not efficient in detecting ESBL-producing E. coli. Similarly, ZOX, CTX, and ATM were efficient in detecting 95\%, 81.7\%, 71.7\% ESBL-producing Klebsiella pneumonia, respectively. The other antibiotics could detect few ESBLproducing $K$. pneumoniae.
In Klebsiella oxytoca, ZOX showed excellent performance by detecting 7/8 (87.5) of the ESBL-producing isolates. ATM detected $75 \%$ of the isolates, while the other antibiotics detected only $50 \%$ or less of the ESBL-production in the isolates.

In Enterobacter cloacae, CTX and CRO performed excellently by detecting 14/15 (93.3\%) of the ESBL producing isolates, whereas CAZ and ZOX detected $13 / 15(86.7 \%)$ of the ESBL producers, while CPD detected only $12 / 15(80 \%)$ of them.

In conclusion, ESBL-producing gram negative bacteria constituted $22.9 \%$ (108/472) of all recovered isolates, and were prevalent among E. coli, K. pneumoniae, K. oxytoca, Enterobacter cloacae, especially from samples collected from patients in secondary and tertiary care hospitals. The inclusion of ceftizoxime (ZOX) to the ESBL-detection panel will increase the efficacy of the DDD test in detecting ESBL producers.

\section{Acknowledgment}

The authors would like to thank the Faculty of Scientific Research at Jordan University of Science and Technology for the financial funding of this study.

\section{References}

[1] R. Bonnet, C. De Champs, D. Sirot, C. Chanal, R. Labia, and J. Sirot, "Diversity of TEM mutants in Proteus mirabilis," Antimicrobial Agents and Chemotherapy, vol. 43, no. 11, pp. 2671-2677, 1999.

[2] G. Arlet, G. Brami, D. Decrere, et al., "Molecular characterization by PCR-restriction fragment length polymorphism of TEM $\beta$-lactamases," FEMS Microbiology Letters, vol. 134, pp. 1498-1500, 1995.

[3] J. Blazquez, M. I. Morosini, M.-C. Negri, and F. Baquero, "Selection of naturally occurring extended-spectrum TEM $\beta$ lactamase variants by fluctuating $\beta$-lactam pressure," Antimicrobial Agents and Chemotherapy, vol. 44, no. 8, pp. 21822184, 2000.

[4] J. Walther-Rasmussen and N. Høiby, "Plasmid-borne AmpC $\beta$-lactamases," Canadian Journal of Microbiology, vol. 48, no. 6, pp. 479-493, 2002.

[5] G. Arlet and A. Philippon, "Construction by polymerase chain reaction and intragenic DNA probes for three main types of transferable $\beta$-lactamases (TEM, SHV, CARB)," FEMS Microbiology Letters, vol. 82, pp. 19-26, 1991.

[6] A. Bauernfeind, I. Stemplinger, R. Jungwirth, S. Ernst, and J. M. Casellas, "Sequences of $\beta$-Lactamase genes encoding CTX-M-1 (MEN-1) and CTX-M-2 and relationship of their amino acid sequences with those of other $\beta$-lactamases," Antimicrobial Agents and Chemotherapy, vol. 40, pp. 509-513, 1996.

[7] W. -L. Yu, Y. -C. Chuang, and J. Walther-Rasmussen, "Extended-spectrum beta-lactamases in Taiwan: epidemiology, detection, treatment and infection control," Journal of Microbiology, Immunology and Infection, vol. 39, no. 4, pp. 264-277, 2006.

[8] S. H. Choi, J. E. Lee, S. J. Park, et al., "Prevalence, microbiology, and clinical characteristics of extended-spectrum 
$\beta$-lactamase-producing Enterobacter spp., Serratia marcescens, Citrobacter freundii, and Morganella morganii in Korea," European Journal of Clinical Microbiology and Infectious Diseases, vol. 26, no. 8, pp. 557-561, 2007.

[9] D. Ivanova, R. Markovska, N. Hadjieva, I. Schneider, I. Mitov, and A. Bauernfeind, "Extended-spectrum $\beta$-lactamaseproducing Serratia marcescens outbreak in a Bulgarian hospital," Journal of Hospital Infection, vol. 70, no. 1, pp. 60-65, 2008.

[10] R. Bonnet, J. L. M. Sampaio, C. Chanal, et al., "A novel class A extended-spectrum $\beta$-lactamase (BES-1) in Serratia marcescens isolated in Brazil," Antimicrobial Agents and Chemotherapy, vol. 44, no. 11, pp. 3061-3068, 2000.

[11] J. J. Jean, P. R. Hsueh, W. S. Lee, et al., "Nationwide surveillance of antimicrobial resistance among Enterobacteriaceae in intensive care units in Taiwan," European Journal of Clinical Microbiology and Infectious Diseases, vol. 28, no. 2, pp. 215220, 2009.

[12] J. Färber, K. A. Moder, F. Layer, I. Tammer, W. König, and B. König, "Extended-spectrum beta-lactamase detection with different panels for automated susceptibility testing and with a chromogenic medium," Journal of Clinical Microbiology, vol. 46, no. 11, pp. 3721-3727, 2008.

[13] K. Bush, G. A. Jacoby, and A. A. Medeiros, "A functional classification scheme for $\beta$-lactamases and its correlation with molecular structure," Antimicrobial Agents and Chemotherapy, vol. 39, no. 6, pp. 1211-1233, 1995.

[14] F. H. M'Zali, A. Chanawong, K. G. Kerr, D. Birkenhead, and P. M. Hawkey, "Detection of extended-spectrum $\beta$-lactamases in members of the family Enterobacteriaceae: comparison of the MAST DD test, the double disk and the Etest ESBL," Antimicrobial Agents and Chemotherapy, vol. 45, pp. 881-885, 2000.

[15] R. A. Bonomo, S. A. Rudin, and D. M. Shlaes, "Tazobactam is a potent inactivator of selected inhibitor-resistant class $\mathrm{A} \beta$ lactamases," FEMS Microbiology Letters, vol. 148, no. 1, pp. 5962, 1997.

[16] P. A. Bradford, Y. Yang, D. Sahm, I. Grope, D. Gardovska, and G. Storch, "CTX-M-5, a novel cefotaxime-hydrolyzing $\beta$ Lactamase from an out-break of Salmonella typhimurium in Latvia," Antimicrobial Agents and Chemotherapy, vol. 42, pp. 1980-1894, 1998.

[17] C. Branger, A. L. Lesimple, B. Bruneau, P. Berry, and N. Lambert-Zechovsky, "Long-term investigation of the clonal dissemination of Klebsiella pneumoniae isolates producing extended-spectrum $\beta$-lactamases in a university hospital," Journal of Medical Microbiology, vol. 47, no. 3, pp. 201-209, 1998.

[18] P. L. Ho, D. N. C. Tsang, T. L. Que, M. Ho, and K. Y. Yuen, "Comparison of screening methods for detection of extended-spectrum $\beta$-lactamases and their prevalence among Escherichia coli and Klebsiella species in Hong Kong," APMIS, vol. 108, no. 3, pp. 237-240, 2000.

[19] H. Pai, S. Lyu, J. H. Lee, J. Kim, Y. Kwon, J.-W. Kim, and K. W. Choe, "Survey of extended-spectrum $\beta$-lactamases in clinical isolates of Escherichia coli and Klebsiella pneumoniae: prevalence of TEM-52 in Korea," Journal of Clinical Microbiology, vol. 37, no. 6, pp. 1758-1763, 1999.

[20] T. Yagi, H. Kruokawa, N. Shibata, K. Shibayama, and Y. Arakawa, "A preliminary survey of extended-spectrum $\beta$ lactamases (ESBLs) in clinical isolates of Klebsiella pneumoniae and Escherichia coli in Japan," FEMS Microbiology Letters, vol. 184, pp. 53-56, 2000.
[21] J. J. Yan, S. M. Wu, S. H. Tsai, J. J. Wu, and I. J. Su, "Prevalence of SHV-12 among clinical isolates of Klebsiella pneumoniae producing extended-spectrum $\beta$-lactamases and identification of a novel AmpC enzyme (CMY-8) in southern Taiwan," Antimicrobial Agents and Chemotherapy, vol. 44, pp. 14381442, 2000.

[22] J. K. Rasheed, C. Jay, B. Metchock, et al., "Evolution of extended-spectrum $\beta$-lactam resistance (SHV-8) in a strain of Escherichia coli during multiple episodes of bacteremia," Antimicrobial Agents and Chemotherapy, vol. 41, pp. 647-653, 1997.

[23] E. W. Koneman, S. D. Allen, W. M. Janda, P. C. Schreckenberger, and W. C. winn, Color Atlas and Textbook of Diagnostic Microbiology, JB Lippincott, Philadelphia, Pa, USA, 4th edition, 1992.

[24] P. R. Murray, E. J. Baron, M. A. Pfaller, et al., Eds., Manual of Clinical Microbiology, American Society for Microbiology, Washington, DC, USA, 7th edition, 1999.

[25] E. J. Baron and S. M. Finegold, "Methods for testing antimicrobial effectiveness," in Bailye Scott's Diagnostic Microbiology, pp. 171-194, The C.V. Mosby Company, St. Louis, Mo, USA, 1990.

[26] D. G. Evans, D. J. Evans, and H. L. DuPont, "Virulence factors of Enterotoxigenic Escherichia coli," The Journal of Infectious Diseases, vol. 136, pp. S118-S123, 1977.

[27] National Committee for Clinical Laboratory Standards, "Methods for dilution antimicrobial susceptibility tests for bacteria that grow aerobically," Approved standard M7-A5 and informational supplement M100-S10, National Committee for Clinical Laboratory Standards, Wayne, Pa, USA, 2000.

[28] V. Jarlier, M. H. Nicolas, G. Fournier, and A. Philippon, "Extended broad-spectrum $\beta$-lactamases conferring transferable resistance to newer $\beta$-lactam agents in Enterobacteriaceae: hospital prevalence and susceptibility patterns," Reviews of Infectious Diseases, vol. 10, no. 4, pp. 867-878, 1988.

[29] M. T. Youssef, H. I. Malkawi, A. A. Shurman, and A. O. Andremont, "Molecular typing of multiresistant Klebsiella pneumoniae isolated from children from northern Jordan," Journal of Tropical Pediatrics, vol. 45, no. 5, pp. 271-277, 1999.

[30] A. A. Shehabia, A. Mahafzah, I. Baadran, F. A. Qadar, and N. Dajani, "High incidence of Klebsiella pneumoniae clinical isolates to extended-spectrum $\beta$-lactam drugs in intensive care units," Diagnostic Microbiology and Infectious Disease, vol. 36, no. 1, pp. 53-56, 2000.

[31] P. A. Bradford, "Extended-spectrum $\beta$-lactamases in the 21st century: characterization, epidemiology, and detection of this important resistance threat," Clinical Microbiology Reviews, vol. 14, no. 4, pp. 933-951, 2001.

[32] T. Spanu, F. Luzzaro, M. Perilli, et al., "Occurrence of extended-spectrum $\beta$-lactamases in members of the family Enterobacteriaceae in Italy: implications for resistance to $\beta$ lactams and other antimicrobial drugs," Antimicrobial Agents and Chemotherapy, vol. 46, no. 1, pp. 196-202, 2002.

[33] H. Wang, S. Kelkar, W. Wu, M. Chen, and J. P. Quinn, “Clinical isolates of Enterobacteriaceae producing extended-spectrum $\beta$ lactamases: prevalence of CTX-M-3 at a hospital in China," Antimicrobial Agents and Chemotherapy, vol. 47, no. 2, pp. 790-793, 2003.

[34] A. A. Kader and K. Angamuthu, "Extended-spectrum $\beta$ lactamases in urinary isolates of Escherichia coli, Klebsiella pneumoniae and other gram-negative bacteria in a hospital in 
Eastern Province, Saudi Arabia," Saudi Medical Journal, vol. 26, no. 6, pp. 956-959, 2005.

[35] E. M. Mokaddas, A. A. Abdulla, S. Shati, and V. O. Rotimi, "The technical aspects and clinical significance of detecting extended-spectrum $\beta$-lactamase-producing Enterobacteriaceae at a tertiary-care hospital in Kuwait," Journal of Chemotherapy, vol. 20, no. 4, pp. 445-451, 2008. 

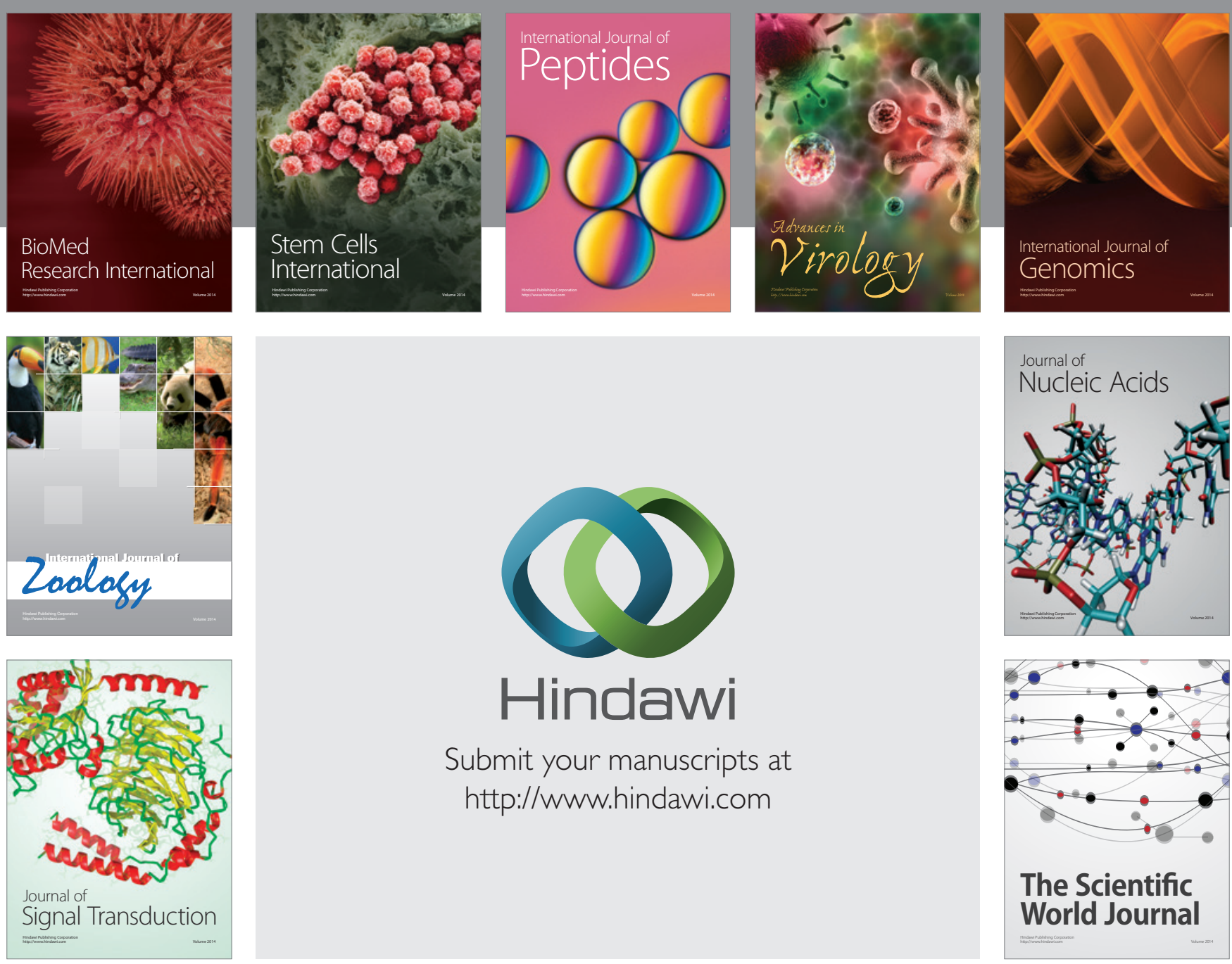

Submit your manuscripts at

http://www.hindawi.com
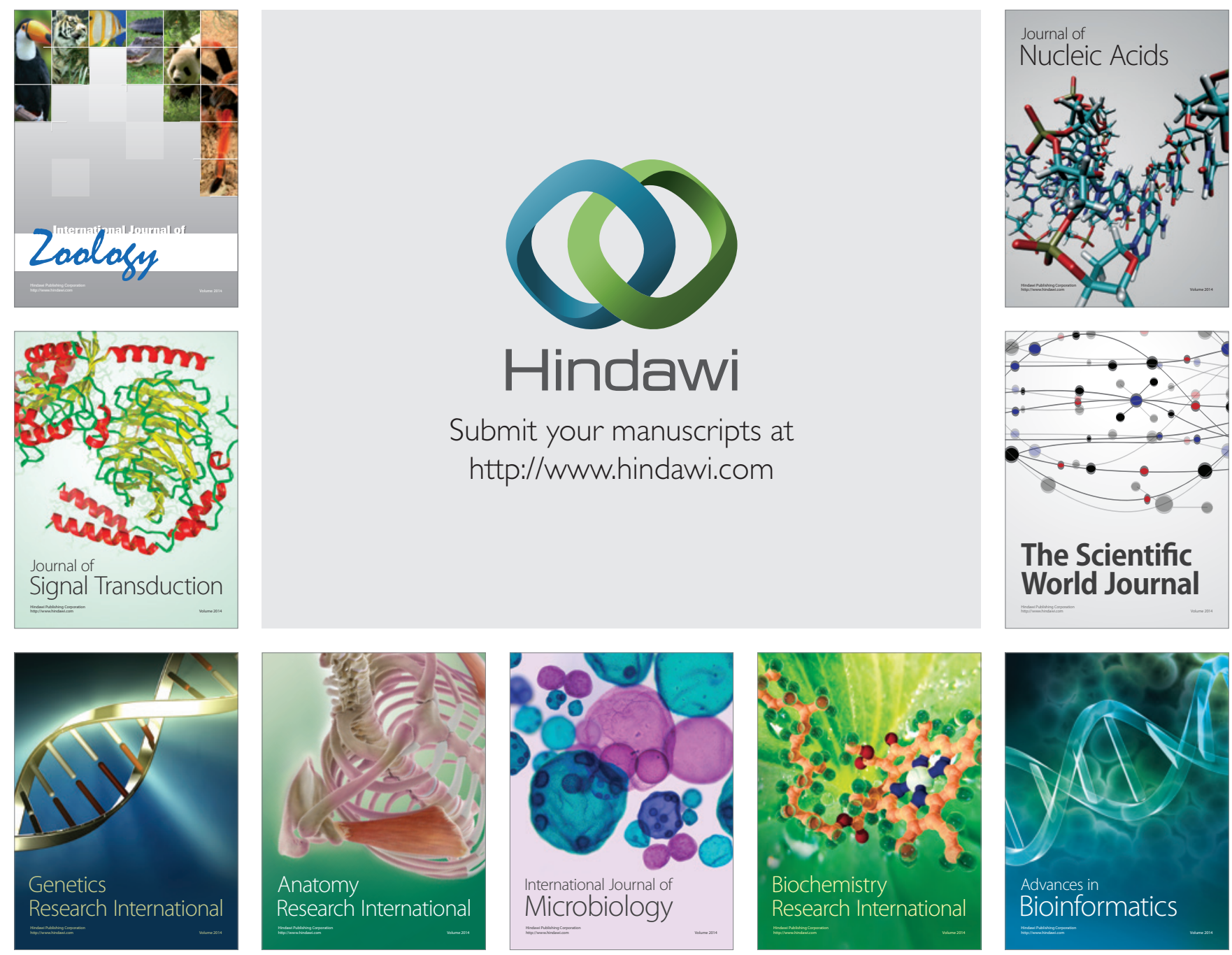

The Scientific World Journal
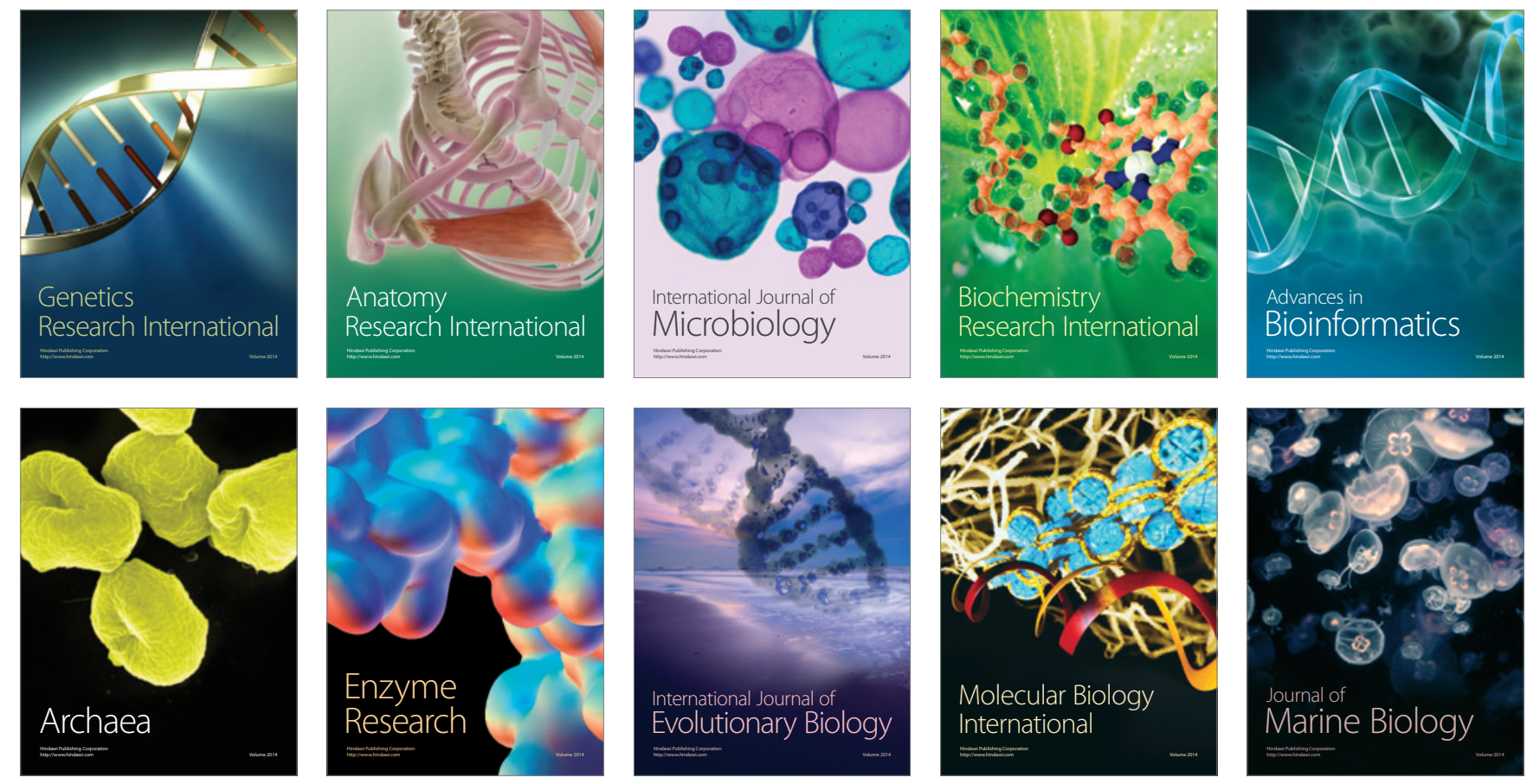